OPEN ACCESS

Edited by:

Xenia Gonda,

Semmelweis University, Hungary

Reviewed by:

Imke Baetens,

Vrije University Brussel, Belgium

Jocelyn Meza,

University of California, Los Angeles,

United States

*Correspondence:

Michael Kaess

michael.kaess@upd.ch orcid.org/0000-0003-0031-7764

Specialty section:

This article was submitted to Child and Adolescent Psychiatry,

a section of the journal

Frontiers in Psychiatry

Received: 17 October 2019 Accepted: 16 September 2020 Published: 04 November 2020

Citation:

Edinger A, Fischer-Waldschmidt G,

Parzer P, Brunner R, Resch F and

Kaess M (2020) The Impact of

Adverse Childhood Experiences on

Therapy Outcome in Adolescents

Engaging in Nonsuicidal Self-Injury.

Front. Psychiatry 11:505661.

doi: 10.3389/fpsyt.2020.505661

\section{The Impact of Adverse Childhood Experiences on Therapy Outcome in Adolescents Engaging in Nonsuicidal Self-Injury}

\author{
Alexandra Edinger ${ }^{1,2}$, Gloria Fischer-Waldschmidt ${ }^{1,2}$, Peter Parzer ${ }^{2}$, Romuald Brunner ${ }^{3}$, \\ Franz Resch ${ }^{2}$ and Michael Kaess ${ }^{1,4 *}$
}

${ }^{1}$ Section for Translational Psychobiology in Child and Adolescent Psychiatry, Clinic of Child and Adolescent Psychiatry, Center for Psychosocial Medicine, University Hospital Heidelberg, Heidelberg, Germany, ${ }^{2}$ Clinic of Child and Adolescent Psychiatry, Center for Psychosocial Medicine, University Hospital Heidelberg, Heidelberg, Germany, ${ }^{3}$ Clinic and Policlinic of Child and Adolescent Psychiatry, Psychosomatics and Psychotherapy, University of Regensburg, Regensburg District Hospital, Regensburg, Germany, ${ }^{4}$ University Hospital of Child and Adolescent Psychiatry and Psychotherapy, University of Bern, Bern, Switzerland

Objective: Nonsuicidal self-injury (NSSI) is a prevalent and clinically significant behavior. There is a substantial association between adverse childhood experiences (ACEs) and NSSI. However, there are no studies investigating the impact of ACEs on NSSI treatment (psychotherapy) outcome. The aim of this secondary analysis of a randomized controlled trial $(\mathrm{RCT})$ on psychotherapy of NSSI was to investigate the relationship between ACEs and treatment outcome in adolescents engaging in NSSI.

Method: A sample of 74 adolescent outpatients engaging in repetitive NSSI (incidents on $\geq 5$ days within the last 6 months) was recruited for a RCT. ACEs were assessed by the Childhood Experience of Care and Abuse (CECA) interview before treatment onset. Based on the CECA, participants were divided in two groups: with a history of ACEs $(n=$ 30 ) and without a history of ACEs $(n=44)$. Frequencies of NSSI, depression, and suicide attempts as well as quality of life were measured at three points in time: before treatment onset (baseline; T0), 4 (T1), and 10 months (T2) after treatment onset.

Results: Both participants with and without ACEs were able to reduce the frequency of NSSI significantly $\left[x^{2}(1)=26.72 ; p<0.001\right]$. Surprisingly, participants with ACEs reached a significantly greater reduction in NSSI frequency within the past 6 months compared to participants without ACEs $\left[\chi^{2}(1)=5.08 ; p=0.024\right]$. There were also substantial and similar improvements regarding depressive symptoms, suicide attempts and quality of life in both groups.

Conclusion: ACEs seem to positively predict treatment response in psychotherapy for adolescent NSSI. This is contrary to prior research suggesting ACE as an unfavorable prognostic factor in the treatment of mental disorders.

Clinical Trial Registration: Short term therapy in adolescents with self-destructive and risk-taking behaviors; http://www.drks.de; DRKS00003605.

Keywords: nonsuicidal self-injury, adverse childhood experiences, treatment outcome, adolescents, psychotherapy 


\section{INTRODUCTION}

Nonsuicidal self-injury (NSSI) is defined "as the deliberate, selfinflicted damage of body tissue without suicidal intent and for purposes not socially or culturally sanctioned" (International Society for the Study of Self-Injury, ISSS). NSSI is categorized as an independent disorder in need of further study in the fifth edition of the Diagnostic and Statistical Manual of Mental Disorders (DSM-5) (1). It is a highly recurrent behavior and peaks in adolescence $(2,3)$. Approximately $17-18 \%$ of adolescents worldwide are affected $(4,5)$. The prevalence rate for repetitive NSSI using the criteria of the DSM-5 ranges between 1.5 and $6.7 \%$ in a recent community study (6). In clinical samples, NSSI is exhibited by $50-60 \%$ of adolescents (7). Although NSSI is associated with a variety of psychiatric disorders, including posttraumatic stress disorder (PTSD) (8) and borderline personality disorder (BPD), it also occurs without any comorbid diagnoses (9).

Nock (10) presented an etiology model to explain the development and maintenance of NSSI. Within the model, he postulates both distal risk factors like specific genetic predispositions for high cognitive and emotional reactivity as well as environmental factors such as childhood maltreatment and hostility/criticism within the familial context. These factors are suggested to result in poor emotion regulation and communication skills, which in turn increase the risk for NSSI (10). The postulated distal risk factors of childhood maltreatment and familial hostility can be summarized under the term "adverse childhood experiences" (ACEs). ACEs refer to distressing and/or traumatic events that occur during childhood, such as abuse, deprivation, and neglect (11). A systematic review consisting of 20 cross-sectional studies found a positive association between childhood maltreatment and NSSI (12). More broadly, ACEs have consistently been identified as significant predictors of NSSI among adolescents from the community (13-15). However, ACEs were also specifically predictive of NSSI within child and adolescent patient samples $(7,16-18)$.

Concerning different types of ACEs, experiences of neglectful or harsh parenting seem to play a most prominent role. Previous studies revealed highest associations for maternal antipathy and neglect (7). In line with these findings, a strong association of increased parental critique and apathy has been shown (19). However, it is important to note that longitudinal studies revealed reciprocal effects between NSSI and parenting, e.g., a significant impact of NSSI on parents' well-being and therefore on their ability to support their children $(20,21)$.

Another study found that only child emotional abuse remained significantly associated with NSSI, when different types of ACEs were analyzed simultaneously (18). Also, Brown et al. (22) found that especially emotional neglect and abuse seem to be important in the etiology of NSSI. A recent meta-analysis showed that childhood maltreatment, but in particular emotional abuse, was associated with NSSI (23). Nonetheless, and besides the importance of those experiences above, sexual abuse has been repeatedly shown to be associated with the development and onset of NSSI $(7,24-26)$.
Not all adolescents with NSSI report a history of ACEs. Previous studies revealed frequencies of $64 \%$ among samples of adolescent inpatients engaging in NSSI (7). Within community samples, $53.3 \%$ of adolescents with NSSI reported ACEs, most frequently emotional abuse (27). Interestingly, the presence of ACEs was significantly related to automatic functions of NSSI (e.g., affect regulation, anti-dissociative function, or selfpunishment) within a study on adolescent inpatients with repetitive NSSI (7). In line with these findings, it was shown that adolescents with greater ACEs showed poorer self-regulation than adolescents without ACEs (28).

There are treatment options which are useful in the treatment of NSSI, like dialectical behavior therapy for adolescents [DBTA $(29,30)]$ and mentalization-based treatment for adolescents [MBT-A (31)]. Recently, our working group evaluated a specific short-term program for adolescent NSSI, which shows to be as effective as treatment as usual in reducing NSSI as well as common comorbid symptomatology (32). However, in terms of a personalized medicine (33), no criteria exist-beyond the presence of NSSI - that may guide adequate decision making regarding which treatment is best for the individual patient. Considering the transdiagnostic character of NSSI (34), more specific indicators are needed to provide individuals with the best-fitting therapy to increase effectiveness. Therefore, studies investigating predictors of treatment outcome are warranted in order to facilitate personalized treatment in the future.

Literature postulates that ACEs have a negative impact on treatment outcome. One idea is that the presence of ACEs leads to more severe psychopathology, which in turn causes poorer prognosis concerning therapy outcomes. A study on depressed outpatients found that those with ACEs showed poorer therapy outcomes: patients with ACEs had a longer time to remission, and they needed a combination treatment of antidepressants and psychotherapy significantly more often compared to their counterparts without ACEs (35). Another idea is that ACEs cause attachment problems, which interfere with the therapeutic alliance (36). The therapeutic alliance is one of the common, unspecific curative factors in psychotherapy (37). Thus, this relationship could explain the poor therapy outcomes in patients with ACEs. Another finding is that ACEs cause severe comorbid psychopathology, which occurs at a later point in time (36). Therefore, there might be unfavorable therapy outcomes because of upcoming psychopathology.

In general, higher numbers of negative life events are associated not only with the onset of psychopathology but also with poorer outcomes and greater chances of relapse (38). A meta-analysis of 16 epidemiological studies suggested that ACEs were associated with an elevated risk of developing persistent and recurrent depressive episodes (39). A meta-analysis of 10 clinical trials revealed that ACEs were associated with lack of response or remission during treatment for depression. It was concluded that ACEs predict an unfavorable course of illness and treatment outcome in depression (39). A study with adult dysthymia patients showed similar results: at a 5-year follow-up, patients with experiences of sexual abuse and poor childhood maternal and paternal relationships showed a lower rate of recovery from dysthymic disorder and higher levels of depression compared to 
participants without ACEs (40). In a study investigating therapy response in substance use disorders, emotional abuse as well as witnessed assaults were negatively related to treatment outcome, whereas physical and sexual abuse were not predictive (41). Another study investigated predictors of therapy outcome in adult outpatient borderline personality disorder (BPD) patients (42). Childhood physical abuse was one of the significant factors that predicted dropout from treatment. Depressive disorders, BPD, and substance use disorders are often comorbid to NSSI, giving a hint that the same might be true for treatment outcomes in NSSI. However, there are no studies to date examining the impact of ACEs on treatment outcome in adolescents engaging in NSSI.

This secondary data analysis of a previously published (32) randomized controlled trial (RCT) on psychotherapy of adolescent NSSI aimed to investigate the impact of ACEs on treatment outcome, which was defined as a reduction in the frequency of NSSI, suicide attempts, a reduction of depressive symptoms, and an increase in quality of life over time. As primary hypothesis, we assumed that adolescents with ACEs would show poorer treatment outcomes regarding NSSI (reduction of NSSI frequencies within the past 6 months) compared to adolescents with no history of ACEs. As secondary hypothesis, we assumed that adolescents with ACEs would show poorer treatment outcomes regarding suicide attempts, depression, and quality of life compared to participants without a history of ACEs.

\section{MATERIALS AND METHODS}

The original RCT evaluated the efficacy of a new cognitivebehavioral short-term program for adolescent NSSI, the CuttingDown Programme [CDP (43)], compared to a high-quality treatment as usual (TAU). The detailed protocol was registered at the German Clinical Trials Register (DRKS00003605; http:// www.drks.de). In addition, study protocol (44) and the results of the original study (32) have been published elsewhere. The present study investigated the impact of ACEs on treatment outcome within this RCT. To test the mentioned hypotheses, a quasi-experimental study with a between-subject design with repeated measures was conducted.

\section{Participants and Procedure}

The study comprised a sample of 74 participants (mean age 14.9 years, $\mathrm{SD}=1.2 ; 96.0 \%$ female) which were recruited through in- and outpatient units at the Clinic of Child and Adolescent Psychiatry at the University Hospital Heidelberg, Germany. Ethical approval was obtained from the institutional review board of the medical faculty at the University of Heidelberg (Ethics Committee No.: S-363/2011). The data analyzed were collected between February 2012 and 2017. Eligible participants were between 12 and 17 years old and were required to have engaged in NSSI on at least 5 days during the past 6 months (DSM5 criterion A). The last incident of NSSI must not have dated back longer than 1 month. Exclusion criteria were as follows: acute psychotic symptoms; acute intent to harm self or others, which required an intensive psychiatric intervention; an impaired intellectual functioning; receiving current psychotherapeutic treatment. Subjects were included into the study only if both adolescents and caregivers had given their written consent. Before, they were informed about the purposes, content as well as risks, and benefits of the study by an information sheet.

Within the original study, participants were randomly assigned to receive on average 10 sessions of CDP or 19 sessions of treatment as usual (TAU). The CDP was delivered according to the manual by therapists in our specialized outpatient clinic (AtR!Sk), whereas TAU was standard care within the existing mental health care system requiring that TAU therapists agree to provide a first appointment and subsequent therapy within two to 4 weeks. TAU was either cognitive-behavioral therapy or depth psychology. Participants within both groups were able to receive general psychosocial management as well as pharmacological treatment, as needed. All study therapists received training in the CDP beforehand. Within the present study, participants were separated in two groups: participants with at least one ACE and participants with no history of ACEs.

Study participants were assessed at multiple time points: before treatment (T0) and four (T1) and 10 months (T2) after the beginning of the treatment. Participants received monetary compensation for participating in each assessment.

\section{Assessment Measures Assessment of ACEs}

ACEs were assessed at T0 using the Childhood Experience of Care and Abuse (CECA) Interview (45), which is considered to be the gold standard criterion in this field of research. It is a semi-structured interview with an investigator-based approach to rating. Instead of the subject's feelings, behavioral indicators of perpetrators' actions are assessed. The core domains are as follows: parental antipathy, parental neglect, physical abuse, sexual abuse, and psychological abuse. The CECA Interview is a reliable measure both in adults and in adolescents. CECA interviewers receive extensive training before being allowed to use the instrument. Inter-rater reliability was satisfactory both in the English and in the German version (original version: $\kappa \mathrm{s}=$ 0.62-1.00; German version: 0.68-1.00) $(45,46)$.

\section{Assessor's Training}

In the context of the present study, the clinical psychologist, who conducted the CECA interview, was intensively trained in assessing the interview beforehand.

Training consisted of different aspects:

1. Training manual: there was a training manual with detailed instructions and guidelines about the conduct of the CECA interview including many examples for practice.

2. Training: the clinical psychologist who assessed the CECA interviews was trained by Antonia Bifulco, who developed the CECA, within a two-day workshop comprising practical exercises and ratings.

\section{Inter-rater Reliability}

To check for inter-rater reliability, 20 (27.0\%) audiotaped CECA interviews of the clinician assessing the CECA were assessed by an independent second rater blind for the first rater's scores. Inter-rater reliability was very good $(\kappa=0.84$ for psychological 
abuse, $\kappa=0.89$ for role reversal, $\kappa=0.89$ for paternal antipathy, and $\kappa=1.00$ for maternal antipathy and neglect, paternal neglect as well as physical and sexual abuse).

\section{Outcome Measures}

NSSI and suicide attempts were assessed with the German version of the Self-Injurious Thoughts and Behaviors Interview (SITBIG) $(47,48)$ at T0, T1, and T2. Common comorbid mental disorders were assessed at T0 using the German version of the Mini-International Neuropsychiatric Interview for children and adolescents (M.I.N.I.-KID 6.0) (49) and parts of the Structured Clinical Interview for DSM-IV-Axis II (SKID-II) (50). Criteria of the following personality disorders were assessed: avoidant, dependent, borderline, and antisocial personality disorder.

In addition, the following self-report measures were used for study assessment at T0, T1, and T2: participants reported on depression symptoms using the German version of the Beck Depression Inventory II (BDI-II) (51). To assess subjective health and well-being at all three evaluations, participants filled out the KIDSCREEN-27 questionnaire (52). For further information on the assessment measures, see the detailed and published original study (32).

\section{Statistical Analysis}

Descriptive analyses were used to characterize the baseline study sample. Nominal data are presented as frequencies, while continuous data are presented as mean and standard deviation (SD). For variables with highly askew distribution, data are presented as medians and interquartile ranges.

The changes in NSSI over time were analyzed with mixedeffect negative binomial regression because of the overdispersion of rates. Changes in depressive symptoms, suicide attempts, and quality of life over time were analyzed with mixed-effect multilevel regression.

A mixed-effect negative binomial regression was calculated to investigate the impact of single forms of ACE on NSSI. Subsequently, a stepwise regression model was conducted in order to minimize the Bayes Information Criterion (BIC). Thus, single ACE forms with lower independent effects on NSSI were gradually taken out of the model. Pearson correlations were calculated to describe the inter-correlations of ACEs (see Supplementary Material). The analyses were performed with Stata (version 15; StataCorp LLC, College Station, TX, USA).

\section{RESULTS}

\section{Prevalence of ACEs and Sociodemographic Characteristics}

Based on the CECA interview, participants were separated in two groups: 30 patients (40.5\%) reported at least one ACE. This compared with 44 participants (59.5\%) with no history of ACEs $(p=0.108)$.

Antipathy was the most common form of ACEs $(n=28$, 93.3\%). Maternal antipathy $(n=17,56.7 \%)$ was more common than paternal antipathy $(n=11,36.7 \%)$ within the ACE group. The second leading form of ACEs was neglect ( $n=16,53.3 \%)$. Here, paternal neglect was more common ( $n=11,36.7 \%)$ than maternal neglect ( $n=5,16.7 \%)$. Detailed information on all ACE frequencies as well as baseline demographic and clinical characteristics regarding the two groups is shown in Table 1. There were no differences in the baseline demographic characteristics and diagnostic variables between the two groups. Table 2 includes all outcome variables at different time points. There was a marginal significant difference concerning NSSI within the past 6 months at T2 between the ACE and no-ACE group. The ACE group showed a marginal significant greater reduction in NSSI frequency $\mathrm{X}$. Concerning depression and quality of life, there were no differences between the groups. Importantly, there was no difference between the ACE and noACE group concerning the use of interventions (number of sessions completed; $p=0.236$ ).

\section{Adverse Childhood Experiences and NSSI}

Regarding NSSI frequencies, both the participants with ACEs and participants without ACEs reached a significant reduction within the past 6 months over time $\left[\chi^{2}{ }_{(1)}=26.72 ; p<0.001\right]$ with a marginal significant difference between the two groups in favor of the ACE group $\left[\chi^{2}{ }_{(1)}=3.70 ; p=0.054\right.$; Table 2]. A significant point of measurement $\times$ ACE interaction $\left[\chi^{2}(1)\right.$ $=5.08 ; p=0.024]$ regarding the frequency of NSSI within the past 6 months was found. Thus, participants with ACEs reached a greater reduction in the frequency of NSSI than participants without ACEs. The course of NSSI frequency is shown in Figure 1. We also investigated the impact of therapy group affiliation, since the participants received either a specific shortterm therapy on NSSI or treatment as usual (32). No interaction with treatment group affiliation was found, indicating that the treatment received did not affect our results. Furthermore, we reanalyzed the data controlling for depression and BPD, which did not change the results. Thus, results without covariates are presented.

To additionally investigate the impact of different types of ACEs on the course of NSSI, we performed a mixedeffect negative binomial regression. Only one form of ACEs, namely, paternal neglect, reached significance for reduction of NSSI frequency within the model $\left[\chi^{2}{ }_{(1)}=13.21 ; p<\right.$ 0.001]. This variable also showed a significant point of time $\times$ paternal neglect interaction $\left[\chi^{2}{ }_{(1)}=4.50 ; p=0.034\right]$. However, performing a stepwise regression, no single form of ACEs remained within the model, suggesting that there is no specific type of ACE that was responsible for the overall effect in this study.

\section{Adverse Childhood Experiences and Suicide Attempts}

A significant reduction of suicide attempts could be reached by both groups $\left[\chi^{2}{ }_{(2)}=12.67 ; p=0.002\right]$. Again, there was no difference between the ACE and no-ACE group $\left[\chi^{2}{ }_{(1)}=3.21\right.$; $p=0.073]$ and no significant point of measurement $\times$ ACE interaction $\left[\chi^{2}(1)=2.95 ; p=0.086\right]$. 
TABLE 1 | Sociodemographic and clinical characteristics of participants by ACEs at TO.

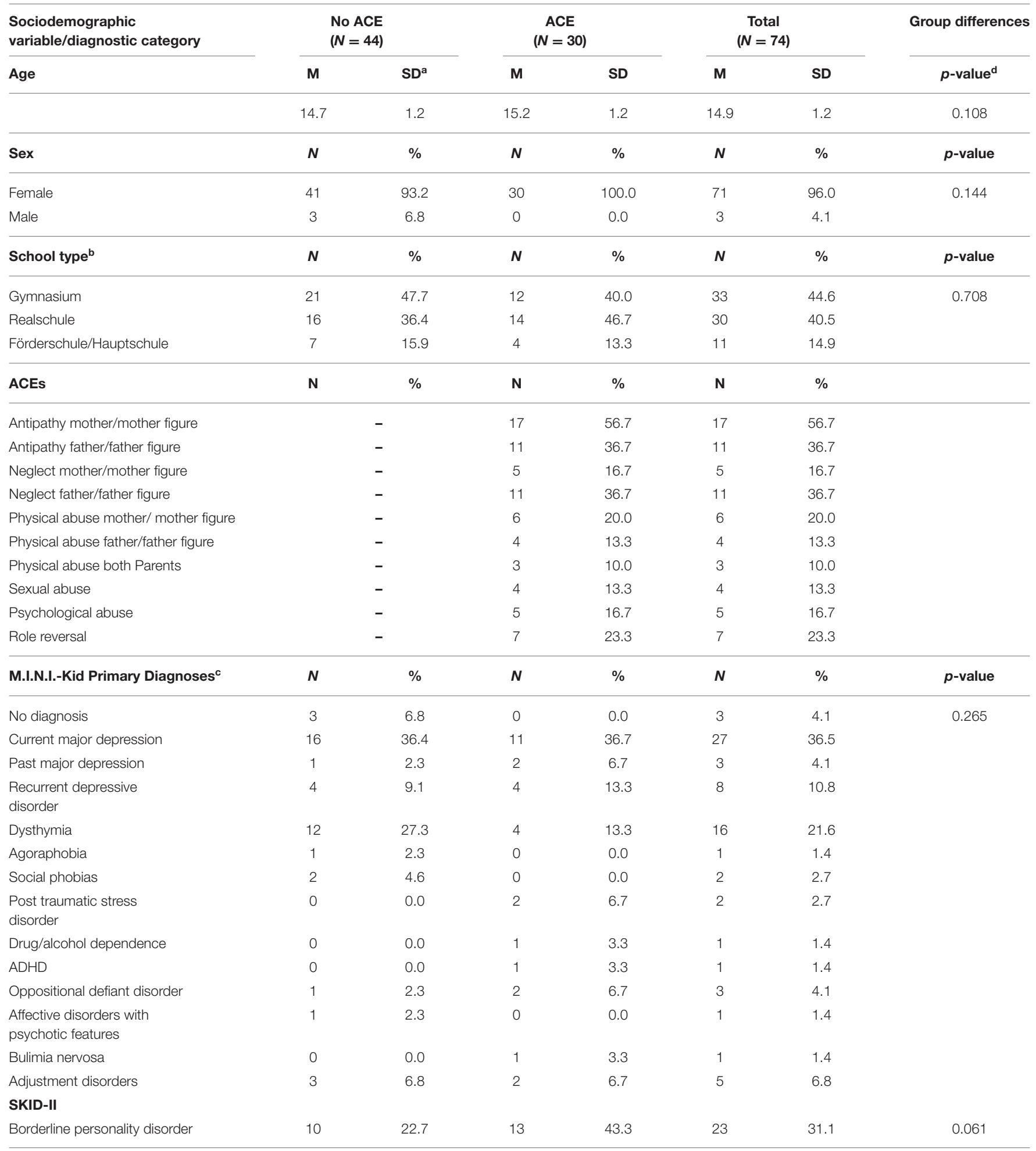

aSD, standard deviation.

${ }^{b}$ Foerderschule, school for students with special needs; Hauptschule, 9 years of elementary school; Realschule, 6 years of school after 4 years of elementary school, terminating with a secondary school level-I certificate; Gymnasium, 8 years of school after 4 years of elementary school, terminating with the general Qualification For University entrance.

${ }^{c}$ Multiple diagnoses per subject possible.

${ }^{d}$ Baseline group differences regarding sociodemographic variables and diagnostic categories. 


\section{Adverse Childhood Experiences and Depression}

Both participants with or without ACEs reached a significant reduction concerning depressive symptoms $\left[\chi^{2}{ }_{(2)}=56.46 ; p<\right.$ $0.001]$ without any difference between the two groups $\left[\chi^{2}{ }_{(1)}=\right.$ $0.00 ; p=0.996$; Table 2] and no significant point of measurement $\times$ ACE interaction $\left[\chi^{2}{ }_{(2)}=1.81 ; p=0.404\right]$.

\section{Adverse Childhood Experiences and Quality of Life}

Concerning quality of life, both groups were able to improve this aspect significantly $\left[\chi^{2}{ }_{(2)}=44.62 ; p<0.001\right]$; however, there was no difference between the two groups $\left[\chi^{2}{ }_{(1)}=0.24 ; p=\right.$ 0.628; Table 2]. Again, no significant point of measurement $x$ $\mathrm{ACE}$ interaction was found $\left[\chi^{2}{ }_{(2)}=2.23 ; p=0.328\right]$.

\section{DISCUSSION}

The purpose of this secondary analysis was to examine the impact of ACEs on therapy outcome within an RCT on adolescent NSSI. According to research, ACEs are a common risk factor for NSSI $(10,53-55)$. In the present study, $40.5 \%(n=30)$ of participants reported a history of ACEs. This prevalence was somewhat smaller than those found in previous studies [64.0\% (7), 79\% (56)]. However, other studies assessed ACEs using questionnaires while the CECA interview was applied in the present study $(45,46)$, which thoroughly assesses behavioral indicators of care and abuse rather than not only the subject's feelings. This standardized and strict procedure might explain the lower prevalence of ACEs in the present study.

The most common forms of ACEs were antipathy, in particular maternal antipathy, and neglect, especially paternal neglect. These findings are in line with previous studies showing that antipathy, neglect, parental critique, apathy, and emotional abuse were highly associated with NSSI $(7,18,19,22,23)$.

Contrary to our hypothesis and contrary to former research (38-41), there was a significant, positive association between ACEs and treatment outcome concerning NSSI frequency. Thus, ACEs were not an unfavorable factor concerning treatment outcome. In fact, the opposite finding emerged. Participants with a history of ACEs showed a greater reduction in NSSI frequency compared to participants without a history of ACEs. Furthermore, both groups reached a significant improvement in suicide attempts, depression, and quality of life with no differences between the two groups. Thus, the ACE group was not inferior to participants without a history of ACEs concerning any other treatment outcomes.

According to the etiology model of Nock (57), which considers the interaction between adverse environmental factors and genetic predisposition, it can be assumed that there might be a stronger impact of biological aspects on NSSI patients without a history of ACEs compared to those with a history of ACEs. Thus, the impact of psychotherapy on the biological vulnerability might be smaller than its impact on environmental factors. These considerations are in line with findings from Nemeroff et al. (58). Traumatized patients with depressive symptoms responded
TABLE 2 | Treatment and clinical outcomes by ACEs.

\begin{tabular}{|c|c|c|c|c|c|}
\hline \multirow{2}{*}{$\begin{array}{l}\text { Intervention/ } \\
\text { clinical outcome } \\
\text { NSSI in last } 6 \text { months }\end{array}$} & \multicolumn{2}{|c|}{ No ACE } & \multicolumn{2}{|c|}{ ACE } & \multirow{2}{*}{$\frac{\text { Group differences }}{p \text {-value }}$} \\
\hline & Median & $I \mathbf{R}^{\mathrm{a}}$ & Median & IQR & \\
\hline TO & 60 & 30-90 & 50 & $20-120$ & 0.054 \\
\hline $\mathrm{T} 1$ & 40 & $15-100$ & 30 & $11-72.5$ & \\
\hline T2 & 13.5 & $1.5-56$ & 4 & $2-13$ & \\
\hline BDI-II scores & $M$ & SD & $M$ & SD & $p$-value \\
\hline TO & 32.0 & 11.2 & 34.0 & 10.6 & 0.996 \\
\hline $\mathrm{T} 1$ & 27.0 & 12.8 & 24.5 & 15.4 & \\
\hline T2 & 21.9 & 14.9 & 21.8 & 13.9 & \\
\hline KIDSCREEN-27 & $M$ & SD & $M$ & SD & $p$-value \\
\hline T0 & 39.4 & 6.2 & 37.3 & 6.1 & 0.489 \\
\hline T1 & 41.7 & 6.6 & 42.1 & 7.6 & \\
\hline T2 & 44.5 & 7.9 & 43.8 & 9.5 & \\
\hline
\end{tabular}

aInterquartile range.

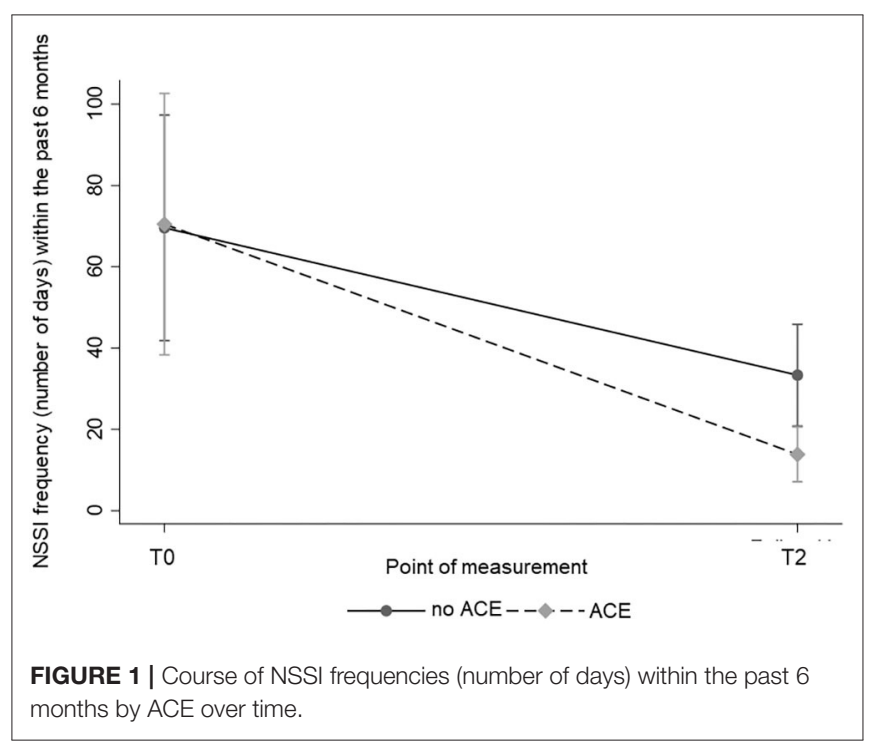

significantly better to a combination of cognitive behavior therapy and pharmacotherapy than to pharmacotherapy alone.

The focus of every treatment applied in the present study (CDP, CBT, etc.) is to learn emotion regulation strategies. These strategies have been reported to be underdeveloped in patients with ACEs compared to patients without ACEs (54). In accordance to these findings, Kaess et al. (7) showed that some forms of ACEs are associated with automatic functions of NSSI, like emotion regulation (59). Thus, there might be a stronger response to these interventions in patients with a history of ACEs.

Similar surprising findings were reported in another study examining BPD patients (60). Patients with less ACEs and a better mother-child relationship reported more suicide attempts than patients with a history of ACEs and a bad motherchild relationship. It was suggested that patients living under good circumstances showed greater hopelessness compared to 
their counterparts because of not performing well in spite of good living conditions. Another study investigating adolescents within a community sample found that those reporting NSSI experienced a significant increase in the quality of relationships with their fathers. This finding offers empirical support for the social positive reinforcement function of NSSI and might add information to the surprising findings (61).

Another reason might be heightened therapy motivation in patients with a history of ACEs. Therapy motivation was found to be an important factor for therapy success across different disorders $(62,63)$. Due to higher psychological strain, participants with ACEs might be more motivated than patients without ACEs. In addition, higher rates of hopelessness in participants without ACEs might decrease therapy motivation in these patients. A recent study in male youth (Mean age: 14.7, $\mathrm{SD}=1.5$ ) with ACEs living in a residential home found that adolescents with four or more ACEs showed higher rates of treatment engagement (64). In addition, Steinke and Derrick (64) found that patients with a history of abuse had higher levels of readiness to change at admission than those with no history of abuse. The same might be true in the present sample.

In addition, the concept of differential susceptibility extends the understanding that negative environments and ACEs exert negative effects, such as poor treatment outcomes, on children or adolescents presumed "environmentally vulnerable." In fact, it reflects that heightened susceptibility to negative effects of ACEs and negative environments may also mean heightened susceptibility to positive and supportive environments (65). Thus, adolescents with a history of ACEs may benefit in particular from caring interactions such as psychotherapeutic interventions. This could explain the faster improvement concerning NSSI in adolescents with ACEs compared to adolescents without ACEs.

With these considerations in mind, it would be helpful to instruct parents to provide more caring interactions, as studies found that perceived family support appears to be an important safeguard against NSSI (66). As a clinical implication, existing treatment approaches should also focus on parents as paternal antipathy and emotional neglect seem to be crucial risk factors for NSSI. To meet this point, our working group started to develop a corresponding manual for parents to enrich the Cutting Down Program. Within DBT-A, participation of parents in group and individual therapy is already a fixed component, which seems to be relevant following existing findings.

\section{Limitations}

The study has several limitations. First, the limited sample size does not allow us to do meaningful differential analyses on type, severity, or chronicity of ACEs. Further research should focus on this point. Moreover, the sample consisted predominantly of female participants, which did not allow drawing conclusions on possible effects for males or gender differences. However, considering that female gender has been identified as a risk factor for NSSI, the presented sample depicts this finding $(3,67)$. Concerning the analyses of single ACE forms, it needs to be considered that the types of ACEs were not equally distributed, which might depict reality on the one hand but generated small subgroups which could have contributed to a lack of significant results on the other hand. Thus, also findings on paternal neglect should be interpreted as explorative, especially since no single ACE remained within the model after stepwise regression. Paternal neglect could be investigated in further studies in particular.

A particular strength of the present study is the participation ratio. There was no dropout from research. Furthermore, the CECA interview was used to assess ACEs. The CECA interview is recognized as the gold standard in this field of research with good reliability and validity (68). Many previous studies solely assessed subjects' feelings by using questionnaires. However, it should be taken into account that the potential risk of a recall bias may still have influenced the present findings.

\section{CONCLUSION}

With these reservations in mind, this study suggests that participants with ACEs showed similar, and in terms of NSSI even greater improvements during psychotherapeutic treatment compared to participants without a history of ACEs. Considering the essential association between ACEs and NSSI, the present findings possess valuable information for practitioners confronted with adolescents engaging in NSSI. In particular, in the context of a personalized medicine, the identification of specific predictors is crucial to increasing treatment effectiveness (33). In this case, adolescents with NSSI and a history of ACEs may be particularly susceptible to psychotherapeutic treatment and do not seem to represent a group of poorer treatment response as initially expected. In contrast, it may rather be those individuals engaging in NSSI despite no history of ACEs (and a potentially higher biological vulnerability) that may require different or additional treatment options. While further exploration of this relationship with larger samples is required, future research should also consider the impact of single forms of ACEs on treatment outcome.

\section{DATA AVAILABILITY STATEMENT}

The raw data supporting the conclusions of this article will be made available by the authors, without undue reservation.

\section{ETHICS STATEMENT}

The studies involving human participants were reviewed and approved by the institutional review board of the medical faculty at the University of Heidelberg (Ethics Committee No.: S-363/2011). Written informed consent to participate in this study was provided by the participants' legal guardian/next of kin.

\section{AUTHOR CONTRIBUTIONS}

AE wrote the first draft. GF-W participated in recruitment and assessment. PP participated in the design of the study and performed the statistical analyses. RB and FR participated in the design of the study and supervised the study procedure. MK was 
responsible for the study design and coordination. All authors revised the article critically and approved the final version of the manuscript.

\section{FUNDING}

This study was funded by the Dietmar Hopp Foundation (grant number 23011188) and sponsored by additional research funds of the Clinic for Child and Adolescent Psychiatry at the University of Heidelberg.

\section{REFERENCES}

1. American Psychiatric Association. Diagnostic and Statistical Manual of Mental Disorders (DSM-5®). American Psychiatric Pub. (2013).

2. Hawton K, Saunders KE, O'Connor RC. Self-harm and suicide in adolescents. Lancet. (2012) 379:2373-82. doi: 10.1016/S0140-6736(12)60322-5

3. Plener PL, Schumacher TS, Munz LM, Groschwitz RC. The longitudinal course of non-suicidal self-injury and deliberate self-harm: a systematic review of the literature. Borderline Personal Disord Emot Dysregul. (2015) 2:2. doi: 10.1186/s40479-014-0024-3

4. Muehlenkamp JJ, Claes L, Havertape L, Plener PL. International prevalence of adolescent non-suicidal self-injury and deliberate self-harm. Child Adolesc Psychiatry Mental Health. (2012) 6:10. doi: 10.1186/1753-2000-6-10

5. Swannell SV, Martin GE, Page A, Hasking P, St John NJ. Prevalence of nonsuicidal self-injury in nonclinical samples: systematic review, metaanalysis and meta-regression. Suicide Life Threat Behav. (2014) 44:273303. doi: $10.1111 /$ sltb. 12070

6. Zetterqvist M. The DSM-5 diagnosis of nonsuicidal self-injury disorder: a review of the empirical literature. Child Adolesc Psychiatry Mental Health. (2015) 9:31. doi: 10.1186/s13034-015-0062-7

7. Kaess M, Parzer P, Mattern M, Plener PL, Bifulco A, Resch F, et al. Adverse childhood experiences and their impact on frequency, severity, and the individual function of nonsuicidal self-injury in youth. Psychiatry Res. (2013) 206:265-72. doi: 10.1016/j.psychres.2012.10.012

8. Gratz KL, Tull MT. Exploring the relationship between posttraumatic stress disorder and deliberate self-harm: the moderating roles of borderline and avoidant personality disorders. Psychiatry Res. (2012) 199:19-23. doi: 10.1016/j.psychres.2012.03.025

9. Andover MS. Non-suicidal self-injury disorder in a community sample of adults. Psychiatry Res. (2014) 219:305-10. doi: 10.1016/j.psychres.2014.06.001

10. Nock MK. Why do people hurt themselves?: new insights into the nature and functions of self-injury. Curr Dir Psychol Sci. (2009) 18:7883. doi: 10.1111/j.1467-8721.2009.01613.x

11. Saul J, Valle LA, Mercy JA, Turner S, Kaufmann R, Popovic T. CDC grand rounds: creating a healthier future through prevention of child maltreatment. Morb Mortal Weekly Rep. (2014) 63:260-3.

12. Serafini G, Canepa G, Adavastro G, Nebbia J, Belvederi Murri M, Erbuto D, et al. The relationship between childhood maltreatment and non-suicidal self-injury: a systematic review. Front Psychiatry. (2017) 8:149. doi: $10.3389 /$ fpsyt.2017.00149

13. Ford JD, Gómez JM. The relationship of psychological trauma and dissociative and posttraumatic stress disorders to nonsuicidal self-injury and suicidality: a review. J Trauma Dissoc. (2015) 16:232-71. doi: 10.1080/15299732.2015.989563

14. Franzke I, Wabnitz P, Catani C. Dissociation as a mediator of the relationship between childhood trauma and nonsuicidal self-injury in females: a path analytic approach. J Trauma Dissoc. (2015) 16:286302. doi: $10.1080 / 15299732.2015 .989646$

15. Smith NB, Kouros CD, Meuret AE. The role of trauma symptoms in nonsuicidal self-injury. Trauma Violence Abuse. (2014) 15:41-56. doi: 10.1177/1524838013496332

16. Baiden P, Stewart SL, Fallon B. The role of adverse childhood experiences as determinants of non-suicidal self-injury among children and adolescents referred to community and inpatient mental health settings. Child Abuse Neglect. (2017) 69:163-76. doi: 10.1016/j.chiabu.2017.04.011

\section{ACKNOWLEDGMENTS}

The authors thank all adolescent patients and therapists for their participation in the study.

\section{SUPPLEMENTARY MATERIAL}

The Supplementary Material for this article can be found online at: https://www.frontiersin.org/articles/10.3389/fpsyt. 2020.505661/full\#supplementary-material

17. Bifulco A, Schimmenti A, Moran P, Jacobs C, Bunn A, Rusu AC. Problem parental care and teenage deliberate self-harm in young community adults. Bull Menninger Clinic. (2014) 78:95-114. doi: 10.1521/bumc.2014.78.2.95

18. Thomassin K, Shaffer A, Madden A, Londino DL. Specificity of childhood maltreatment and emotion deficit in nonsuicidal selfinjury in an inpatient sample of youth. Psychiatry Res. (2016) 244:103-8. doi: 10.1016/j.psychres.2016.07.050

19. Tschan T, Schmid M, In-Albon T. Parenting behavior in families of female adolescents with nonsuicidal self-injury in comparison to a clinical and a nonclinical control group. Child Adolesc Psychiatry Mental Health. (2015) 9:17. doi: 10.1186/s13034-015-0051-x

20. Arbuthnott AE, Lewis SP. Parents of youth who self-injure: a review of the literature and implications for mental health professionals. Child Adolesc Psychiatry Mental Health. (2015) 9:35. doi: 10.1186/s13034-0150066-3

21. Waals L, Baetens I, Rober P, Lewis S, Van Parys H, Goethals ER, et al. The NSSI family distress cascade theory. Child Adolesc Psychiatry Mental Health. (2018) 12:52. doi: 10.1186/s13034-018-0259-7

22. Brown RC, Heines S, Witt A, Braehler E, Fegert JM, Harsch D, et al. The impact of child maltreatment on non-suicidal self-injury: data from a representative sample of the general population. BMC Psychiatry. (2018) 18:181. doi: 10.1186/s12888-018-1754-3

23. Liu RT, Scopelliti KM, Pittman SK, Zamora AS. Childhood maltreatment and non-suicidal self-injury: a systematic review and meta-analysis. Lancet Psychiatry. (2018) 5:51-64. doi: 10.1016/S2215-0366(17)30469-8

24. Chaplo SD, Kerig PK, Bennett DC, Modrowski CA. The roles of emotion dysregulation and dissociation in the association between sexual abuse and self-injury among juvenile justice-involved youth. J Trauma Dissoc. (2015) 16:272-85. doi: 10.1080/15299732.2015.989647

25. Isohookana R, Riala K, Hakko H, Räsänen P. Adverse childhood experiences and suicidal behavior of adolescent psychiatric inpatients. Eur Child Adolesc Psychiatry. (2013) 22:13-22. doi: 10.1007/s00787-012-0311-8

26. Maniglio R. The role of child sexual abuse in the etiology of suicide and non-suicidal self-injury. Acta Psychiatr Scand. (2011) 124:30-41. doi: 10.1111/j.1600-0447.2010.01612.x

27. Whitlock J. Self-injurious behaviors in a college population. Pediatrics. (2006) 117:1939-48. doi: 10.1542/peds.2005-2543

28. Lackner CL, Santesso DL, Dywan J, O'Leary DD, Wade TJ, Segalowitz SJ. Adverse childhood experiences are associated with self-regulation and the magnitude of the error-related negativity difference. Biol Psychol. (2018) 132:244-51. doi: 10.1016/j.biopsycho.2018.01.006

29. Buerger A, Fischer-Waldschmidt G, Hammerle F, Auer KV, Parzer P, Kaess M. Differential change of borderline personality disorder traits during dialectical behavior therapy for adolescents. J Pers Disord. (2019) 33:11934. doi: 10.1521/pedi_2018_32_334

30. Mehlum L, Tørmoen AJ, Ramberg M, Haga E, Diep LM, Laberg S, et al. Dialectical behavior therapy for adolescents with repeated suicidal and selfharming behavior: a randomized trial. J Am Acad Child Adolesc Psychiatry. (2014) 53:1082-91. doi: 10.1016/j.jaac.2014.07.003

31. Rossouw TI, Fonagy P. Mentalization-based treatment for self-harm in adolescents: a randomized controlled trial. J Am Acad Child Adolesc Psychiatry. (2012) 51:1304-13.e3. doi: 10.1016/j.jaac.2012.09.018

32. Kaess M, Edinger A, Fischer-Waldschmidt G, Parzer P, Brunner R, Resch F. Effectiveness of a brief psychotherapeutic intervention compared with treatment as usual for adolescent nonsuicidal self-injury: a single-centre, 
randomised controlled trial. Eur Child Adolesc Psychiatry. (2020) 29:881-91. doi: 10.1007/s00787-019-01399-1

33. Cuijpers P, Ebert DD, Acarturk C, Andersson G, Cristea IA. Personalized psychotherapy for adult depression: a meta-analytic review. Behav Therapy. (2016) 47:966-80. doi: 10.1016/j.beth.2016.04.007

34. Brunner R, Kaess M, Parzer P, Fischer G, Carli V, Hoven CW, et al. Lifetime prevalence and psychosocial correlates of adolescent direct self-injurious behavior: A comparative study of findings in 11 European countries. J Child Psychol Psychiatry. (2014) 55:337-48. doi: 10.1111/jcpp.12166

35. Miniati M, Rucci P, Benvenuti A, Frank E, Buttenfield J, Giorgi G, et al. Clinical characteristics and treatment outcome of depression in patients with and without a history of emotional and physical abuse. J Psychiatr Res. (2010) 44:302-9. doi: 10.1016/j.jpsychires.2009.09.008

36. Vermetten E, Spiegel D. Trauma and dissociation: implications for borderline personality disorder. Curr Psychiatry Rep. (2014) 16:434. doi: 10.1007/s11920-013-0434-8

37. Xu H, Tracey TJ. Reciprocal influence model of working alliance and therapeutic outcome over individual therapy course. Couns Psychol. (2015) 62:351-9. doi: 10.1037/cou0000089

38. Cassels M, Wilkinson P. Non-suicidal self-injury in adolescence. Paediatr Child Health. (2016) 26:554-8. doi: 10.1016/j.paed.2016.08.006

39. Nanni V, Uher R, Danese A. Childhood maltreatment predicts unfavorable course of illness and treatment outcome in depression: a meta-analysis. Am J Psychiatry. (2012) 169:141-51. doi: 10.1176/appi.ajp.2011.11020335

40. Hayden EP, Klein DN. Outcome of dysthymic disorder at 5-year follow-up: the effect of familial psychopathology, early adversity, personality, comorbidity, chronic stress. Am J Psychiatry. (2001) 158:1864-70. doi: 10.1176/appi.ajp.158.11.1864

41. Ford JD, Hawke J, Alessi S, Ledgerwood D, Petry N. Psychological trauma and PTSD symptoms as predictors of substance dependence treatment outcomes. Behav Res Therapy. (2007) 45:2417-31. doi: 10.1016/j.brat.2007.04.001

42. Arntz A, Stupar-Rutenfrans S, Bloo J, van Dyck R, Spinhoven P. Prediction of treatment discontinuation and recovery from Borderline Personality Disorder: Results from an RCT comparing Schema Therapy and Transference Focused Psychotherapy. Behav Res Ther. (2015) 74:60-71. doi: 10.1016/j.brat.2015.09.002

43. Taylor LMW, Oldershaw A, Richards C, Davidson K, Schmidt U, Simic M. Development and pilot evaluation of a manualized cognitive-behavioural treatment package for adolescent self-harm. Behav Cogn Psychother. (2011) 39:619-25. doi: 10.1017/S1352465811000075

44. Fischer G, Brunner R, Parzer P, Resch F, Kaess M. Short-term psychotherapeutic treatment in adolescents engaging in nonsuicidal self-injury: a randomized controlled trial. Trials. (2013) 14:294. doi: 10.1186/1745-6215-14-294

45. Bifulco A, Brown GW, Harris TO. Childhood experience of care and abuse (CECA): a retrospective interview measure. J Child Psychol Psychiatry. (1994) 35:1419-35. doi: 10.1111/j.1469-7610.1994.tb01284.x

46. Kaess M, Parzer P, Mattern M, Resch F, Bifulco A, Brunner R. Childhood experiences of care and abuse (CECA). Z Kinder Jugendpsychiatr Psychother. (2011) 39:243-52. doi: 10.1024/1422-4917/a000115

47. Fischer G, Ameis N, Parzer P, Plener PL, Groschwitz R, Vonderlin E, et al. The German version of the self-injurious thoughts and behaviors interview (SITBI-G): a tool to assess non-suicidal self-injury and suicidal behavior disorder. BMC Psychiatry. (2014) 14:265. doi: 10.1186/s12888-014-0265-0

48. Nock MK, Holmberg EB, Photos VI, Michel BD. Self-injurious thoughts and behaviors interview: development, reliability, and validity in an adolescent sample. Psychol Assess. (2007) 19:309-17. doi: 10.1037/1040-3590.19.3.309

49. Sheehan DV, Lecrubier Y, Sheehan KH, Amorim P, Janavs J, Weiller E, et al. The Mini-International Neuropsychiatric Interview (M.I.N.I.): the development and validation of a structured diagnostic psychiatric interview for DSM-IV and ICD-10. J Clin Psychiatry. (1998) 59(Suppl.) (1998) 20:2233;quiz 34-57.

50. Fydrich T, Renneberg B, Schmitz B, Wittchen, H-U. SKID II. Strukturiertes Klinisches Interview für DSM-IV, Achse II: Persönlichkeitsstörungen. In: First $\mathrm{MB}$, Spitzer RL, Gibbon M, Williams JBW, Benjamin L, editors. Interviewheft. Eine deutschspeachige, erw. Bearb. d. amerikanischen Originalversion d. SKIDII von (Version 3/96) Göttingen: Hogrefe (1997) 36 p.
51. Beck AT, Steer RA, Brown GK. Beck-Depressions-Inventar (2. Auflage). Frankfurt: Harcourt Test Service GmbH (2006).

52. Ravens-Sieberer U, Gosch A, Erhart M, von Rueden U. The KIDSCREEN Group Europe: The KIDSCREEN Questionnaires, 1st ed. Lengerich: Pabst Science Publisher (2006).

53. Lang CM, Sharma-Patel K. The relation between childhood maltreatment and self-injury: a review of the literature on conceptualization and intervention. Trauma Violence Abuse. (2011) 12:23-37. doi: 10.1177/1524838010386975

54. Muehlenkamp JJ, Kerr PL, Bradley AR, Adams Larsen M. Abuse subtypes and nonsuicidal self-injury: preliminary evidence of complex emotion regulation patterns. J Nerv Mental Dis. (2010) 198:258-63. doi: 10.1097/NMD.0b013e3181d612ab

55. Yates TM, Carlson EA, Egeland B. A prospective study of child maltreatment and self-injurious behavior in a community sample. Dev Psychopathol. (2008) 20:651-71. doi: 10.1017/S0954579408000321

56. Gratz KL, Conrad SD, Roemer L. Risk factors for deliberate selfharm among College Students. Am J Orthopsychiatry. (2002) 72:12840. doi: $10.1037 / 0002-9432.72 .1 .128$

57. Nock MK. Self-Injury. Annu Rev Clin Psychol. (2010) 6:33963. doi: 10.1146/annurev.clinpsy.121208.131258

58. Nemeroff CB, Heim CM, Thase ME, Klein DN, Rush AJ, Schatzberg AF, et al. Differential responses to psychotherapy versus pharmacotherapy in patients with chronic forms of major depression and childhood trauma. Proc Natl Acad Sci U S A. (2003) 100:14293-6. doi: 10.1073/pnas.2336126100

59. Nock MK, Prinstein MJ. A functional approach to the assessment of self-mutilative behavior. J Consult Clin Psychol. (2004) 72:885-90. doi: 10.1037/0022-006X.72.5.885

60. Paris J, Nowlis D, Brown R. Developmental factors in the outcome of borderline personality disorder. Compr Psychiatry. (1988) 29:14750. doi: 10.1016/0010-440X(88)90007-7

61. Hilt LM, Nock MK, Lloyd-Richardson EE, Prinstein MJ. Longitudinal study of nonsuicidal self-injury among young adolescents: rates, correlates, and preliminary test of an interpersonal model. J Early Adolesc. (2008) 28:45569. doi: 10.1177/0272431608316604

62. Keithly LJ, Samples SJ, Strupp HH. Patient motivation as a predictor of process and outcome in psychotherapy. Psychother Psychosom. (1980) 33:8797. doi: $10.1159 / 000287417$

63. Ryan RM, Plant RW, O’Malley S. Initial motivations for alcohol treatment: relations with patient characteristics, treatment involvement, and dropout. Addict Behav. (1995) 20:279-97. doi: 10.1016/0306-4603(94)00072-7

64. Steinke CM, Derrick RM. An exploration of the role of adverse childhood experiences (ACEs) on youth engagement in residential treatment. Child Youth Serv Rev. (2018) 89:355-63. doi: 10.1016/j.childyouth.2018.04.039

65. Belsky J, Bakermans-Kranenburg MJ, van IJzendoorn MH. For better and for worse: differential susceptibility to environmental influences. Curr Dir Psychol Sci. (2007) 16:300-4. doi: 10.1111/j.1467-8721.2007.00525.x

66. Tatnell R, Kelada L, Hasking P, Martin G. Longitudinal analysis of adolescent NSSI: the role of intrapersonal and interpersonal factors. J Abnorm Child Psychol. (2014) 42:885-96. doi: 10.1007/s10802-013-9837-6

67. Bresin K, Schoenleber M. Gender differences in the prevalence of nonsuicidal self-injury: a meta-analysis. Clin Psychol Rev. (2015) 38:5564. doi: 10.1016/j.cpr.2015.02.009

68. Thabrew H, de Sylva S, Romans S. Evaluating childhood adversity. In: Fava GA, Sonino N, Wise TN, editors. The Psychosomatic Assessment, Vol. 32. Basel: Karger Publishers (2012). pp. 35-57.

Conflict of Interest: The authors declare that the research was conducted in the absence of any commercial or financial relationships that could be construed as a potential conflict of interest.

Copyright (๑) 2020 Edinger, Fischer-Waldschmidt, Parzer, Brunner, Resch and Kaess. This is an open-access article distributed under the terms of the Creative Commons Attribution License (CC BY). The use, distribution or reproduction in other forums is permitted, provided the original author(s) and the copyright owner(s) are credited and that the original publication in this journal is cited, in accordance with accepted academic practice. No use, distribution or reproduction is permitted which does not comply with these terms. 